\title{
The local earth magnetic field changes impact on weekly hospitalization due to unstable angina pectoris
}

\author{
Greta Žiubrytė , Gediminas Jaruševičius², Mantas Landauskas ${ }^{3}$, Rollin McCraty ${ }^{4}$, \\ Alfonsas Vainoras ${ }^{5}$ \\ ${ }^{1}$ Faculty of Medicine, Academy of Medicine, Lithuanian University of Health Sciences, Kaunas, Lithuania \\ ${ }^{2}$ Department of Cardiology, Hospital of Lithuanian University of Health Sciences Kaunas Clinics, \\ Kaunas, Lithuania \\ ${ }^{2}$ Cardiology Institute, Lithuanian University of Health Sciences, Kaunas, Lithuania \\ ${ }^{3}$ Department of Mathematical Modelling, Kaunas University of Technology, Kaunas, Lithuania \\ ${ }^{4}$ HeartMath Institute, California, USA \\ ${ }^{5}$ Sport Institute, Lithuanian University of Health Sciences, Kaunas, Lithuania \\ ${ }^{1}$ Corresponding author \\ E-mail: ${ }^{1}$ greta.ziubryte@gmail.com, ${ }^{2}$ gedijaru@yahoo.com, ${ }^{3}$ mantas.landauskas@ktu.lt, \\ rollin@heartmath.org, ${ }^{5}$ rollin@heartmath.org
}

Received 7 March 2018; received in revised form 11 June 2018; accepted 19 June 2018 DOI https://doi.org/10.21595/chs.2018.20020

Check for updates

Copyright (C) 2018 Greta Žiubryte, et al. This is an open access article distributed under the Creative Commons Attribution License, which permits unrestricted use, distribution, and reproduction in any medium, provided the original work is properly cited.

\begin{abstract}
Objectives: Acute coronary syndrome as an acute oxygenated blood deprivation to the heart muscle due to atherosclerotic plaque rupture in the coronary artery followed by thrombosis is possibly associated with changes in the Earth's local time varying magnetic field as they strongly influence hormonal and other regulatory systems' activity. This study analyses the correlation between prevalence of the acute coronary syndrome and the changes in the local time varying aspects of the magnetic field. Methods: Seven-hundred patients admitted to Cardiology Department of Hospital of Lithuanian University of Health Sciences Kaunas Clinics within 2016 due to acute coronary syndrome were retrospectively included into the study. The number of cases per week was compared with the weekly changes of the local Earth magnetic field. The one-year period was divided into two periods: the first-half of the year (weeks 1 to 26) and the second-half of the year (weeks 27 to 52) and more detailed analyses were performed accordingly to the significance of the left main artery lesion. Mean power of local magnetic field fluctuations in Lithuania, measured in pT2 in five different frequency ranges where overlaps between the Schumann resonance and EEG frequency ranges (named as SDelta $(0-3.5 \mathrm{~Hz})$, STheta $(3.5-7 \mathrm{~Hz})$, SAlpha $(7-15 \mathrm{~Hz})$, SBeta $(15-32 \mathrm{~Hz})$ and SGamma $(32-66 \mathrm{~Hz})$ to distinguish them from the EEG bands). Results: Statistically significant weak and moderate correlations between weekly prevalence of acute coronary syndrome admissions and the magnetic field intensities changes were found. Higher intensities in the SBeta and SGamma ranges were associated with a higher number of admissions throughout the year in females and the SGamma range was associated with higher number of admission only during the second-half of the year in males. A higher intensity magnetic field in SDelta, STheta, SAlpha and SBeta ranges was associated with a higher admissions number due to left main artery lesions in males, while a higher intensity in the SGamma range was associated with higher number of admissions due to left main artery lesions in females through the year. Conclusion: Significant correlation between acute coronary syndrome and changes in the local Earth time varying magnetic field intensities was found. Some frequency ranges are associated with an episode of an acute coronary syndrome. Left main artery lesions significantly correlated with magnetic field changes in most of the frequency ranges in males while only one of the frequency ranges in females.
\end{abstract}

Keywords: acute coronary syndrome, unstable angina pectoris, the local Earth magnetic field.

\section{Nomenclature}

Acute coronary syndrome 


$\begin{array}{ll}\text { AO } & \text { Arctic oscillation } \\ \text { CA } & \text { Coronary artery } \\ \text { CAG } & \text { Coronary artery angiography } \\ \text { ECG } & \text { Electrocardiogram } \\ \text { EEG } & \text { Electroencephalogram } \\ \text { LAD } & \text { Left anterior descending artery } \\ \text { LCA } & \text { Left coronary artery } \\ \text { LMA } & \text { Left main artery } \\ \text { MF } & \text { Magnetic field } \\ \text { PDS } & \text { Power spectral density } \\ \text { RCA } & \text { Right coronary artery } \\ \text { RCx } & \text { Circumflex artery } \\ \text { TVMF } & \text { Local Earths time varying magnetic field }\end{array}$

\section{Introduction}

Acute coronary syndrome (ACS) as an acute oxygenated blood deprivation to the heart muscle due to mainly atherosclerotic plaque rupture in the coronary artery (CA) followed by thrombosis $[1,2]$ is one of the leading cardiac cause of hospital admission worldwide. The blood rheological properties are relevant in the pathogenesis of thrombosis manifesting as ACS. Recent studies have indicated that human hormonal regulation differs in the first and the second half of the year which are correlated with changes in the time varying local Earth magnetic field strength (Fig. 1) [3]. To understand the process more completely, it is necessary to focus on possible changes of cellular and molecular regulation in accordance to local Earth time varying magnetic field (TVMF) strength changes. It is already known that isolated animal cells have specific sensitivity to the strength and frequency of fluctuation of the TVMF [4-6]. The TVMF regulatory effect proposed to be associated with the ion cyclotron resonance mechanism [5] whose effect on extracted myocardial cells has been recently demonstrated under laboratory conditions [7]. Scientists have shown that healthy people in isolation from the magnetic field (MF) significantly increases their capillary blood flow and reduces the diastolic blood pressure [8]. Several laboratory studies have demonstrated significant negative correlations between baroreflex sensitivity and MF changes [9]. The reduction of baroreflex sensitivity may lead to higher mortality after ACS episode [10]. Even more, the correlations between strong TVMF and melatonin level reduction have been demonstrated $[11,12]$. It is already known, that higher level of melatonin is associated with better myocardial microcirculation [13].

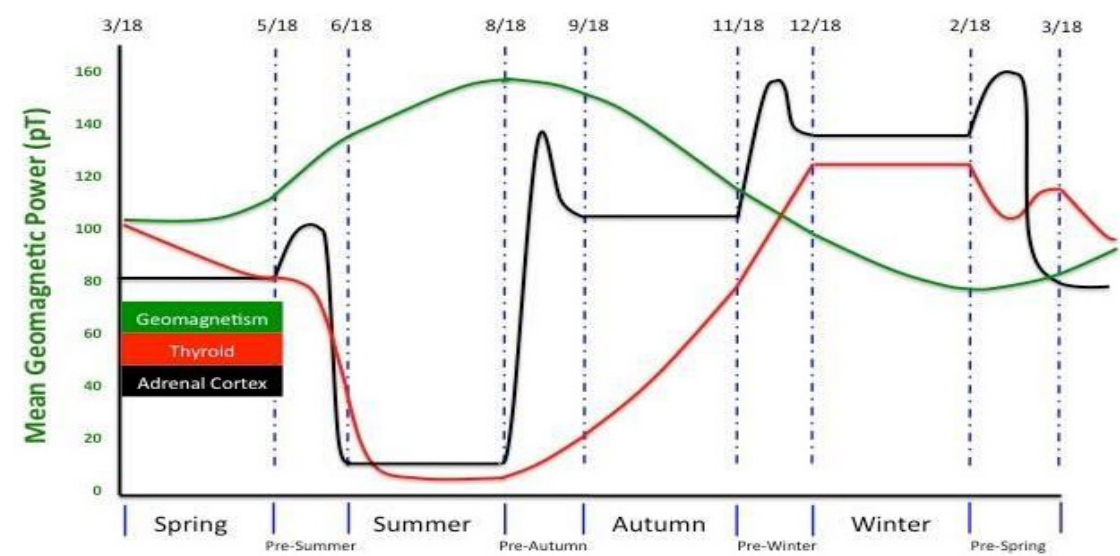

Fig. 1. Relationship between changes of human hormonal activity and the strength of the local Earth magnetic field. [3], with permission from Kamyar Hedayat 
The TVMF is constantly changing and these changes differ within seasons. In winter the TVMF is at its lowest point, start to increase in spring and reaches its peak in the middle-end of summer. The strength of the TVMF abruptly decreases in the autumn to its lowest point at the middle of winter. These changes of the TVMF strength are shown in Fig. 2.

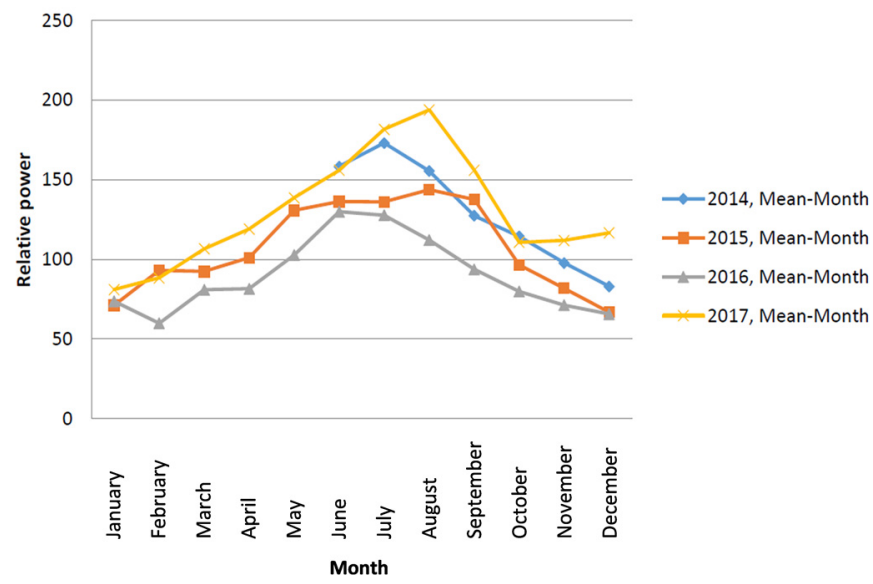

Fig. 2. The changes of strength of the earth's local time varying magnetic field in Lithuania between 2014 June and 2017 December [4]

It is already shown that the TVMF in different frequency intervals has different effect on human regulatory systems. It is believed that low frequency TVMF has positive effect on humans, while high frequency TVMF is recognized as stressful factor to human regulatory systems [13, 14]. Hardelet al. [13] and Huss et al. [15] have shown that both weak and strong MF is associated with negative effect on human health. Due to that, correlations between all frequency ranges and hospital admission were observed in this study. It is interesting that the peaks of the MF spectrum fluctuate at the same range as the human brain activity on electroencephalogram (EEG). The overlapping of Schumann resonance frequencies and EEC waves are presented in Table 1.

Table 1. Overlap between Schumann resonance frequencies and electroencephalogram ranges

\begin{tabular}{|c|c|c|c|c|c|c|}
\hline $\begin{array}{c}\text { Schumann } \\
\text { resonances }\end{array}$ & SDelta & STheta & SAlpha & SBeta & SGamma & $\begin{array}{c}\text { Overall observed MF } \\
\text { spectrum }\end{array}$ \\
\hline EEG ranges, $\mathrm{Hz}$ & $0-3.5$ & $3.5-7$ & $7-15$ & $15-32$ & $32-65$ & $0-65$ \\
\hline
\end{tabular}

Most published studies were focused on the impact of the Global Earth magnetic field changes on human regulatory systems and only a few ones analysed the TVMF effect on human regulatory systems [3]. An acute myocardial infarction association with the TVMF changes were analysed recently [4], but none of studies have investigated the TVMF correlation with cases of unstable angina pectoris yet.

\section{Methods}

\subsection{Study population}

In total $700(425(60.7 \%)$ male and $275(39.3 \%)$ female) patients were admitted to the Cardiology Department of Hospital of Lithuanian University of Health Sciences Kaunas Clinics due to unstable angina pectoris between 1 January 2016 and 31 December 2016 who were retrospectively included in the study. The mean age of males was $65.5( \pm 11.3)$ years and 73.1 $( \pm 9.9)$ years for females. The male group was almost 8 years younger than female group, but no other differences between male and female groups were observed. The number of ACS cases per 
week in male and female is shown in Fig. 3. Patients' baseline characteristics were collected from medical records, after patients' written consent was obtained. Local Ethical committee approval was given. Due to the relatively low number of patients, we did not divided patients into groups according to the age.

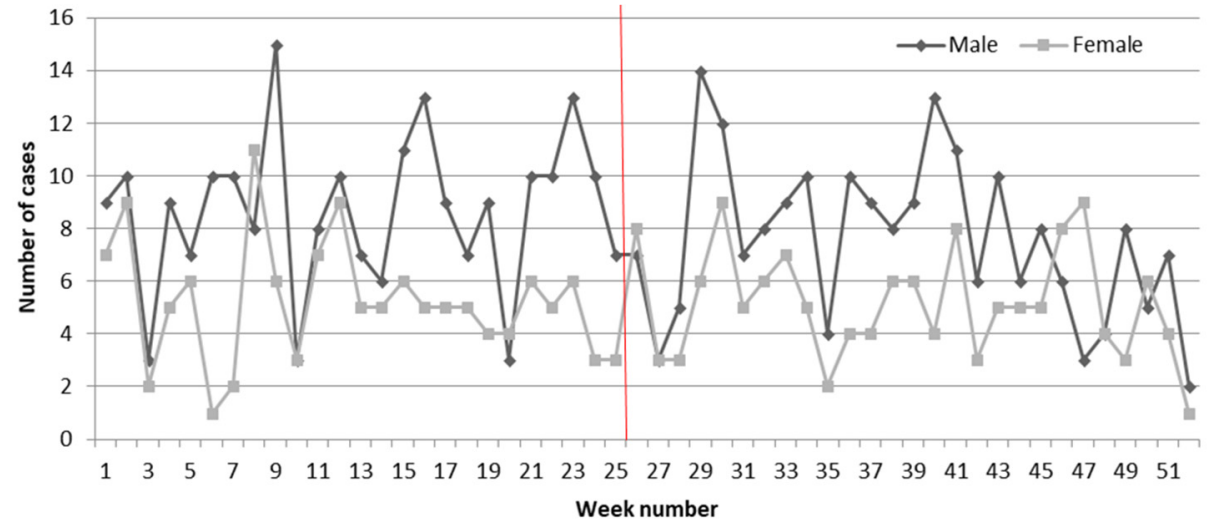

Fig. 3. Number of ACS cases per week in male and female. Red line separates the first (week 1 to 26) and the second half (week 27 to 52) of the year

\subsection{Study and analyses time intervals}

The one-year period was divided into two periods: the first-half of the year (weeks 1 to 26) and the second-half of the year (weeks 27 to 52). We divided the time period due to different activity of the TVMF and the human hormonal regulation in the first and the second halves of the year.

\subsection{Inclusion and exclusion criteria}

All patients admitted to Department of Cardiology in 2016 due to ACS revealed by clinical manifestation, ischemic changes on electrocardiogram (ECG) and increased myocardial necrosis enzymes (Troponin I) were included into the study. For all patients, coronary artery angiography (CAG) was performed. Patients with not performed CAG and/or not elevated Troponin I were excluded.

\subsection{Coronary artery angiography estimation}

The CAGs were analysed in 15 segment model: right coronary artery (RCA) was divided as follow - proximal RCA (S1), mid RCA (S2), distal RCA (S3) and ramus interventricularis posterior (S4); left coronary artery (LCA) was divided into left main artery (LMA) (S5), left anterior descending (LAD), which further divided into five segments - proximal LAD (S6), mid LAD (S7), distal LAD (S8), the first (S9) and the second (S10) diagonal branches; and circumflex artery (RCx) which contains proximal RCx (S11), mid RCx (S13) and the first (S12), the second (S14) and the third (S15) marginal branches. In analyses we merged segments into RCA, LMA, LAD and RCx. CA lesions were considered significant when the diameter stenoses appear more than $50 \%$ by visual estimation.

\subsection{Magnetometer data}

The TVMF intensity was measured by the Global Coherence Monitoring Network magnetometer located In Lithuania. The MF was observed in two (north/south and east/ west) orientations. For analyses in this study we used data from east/west detector following the Heart Math Institute recommendations as this MF direction stronger influence the human health 
conditions. Signals from the magnetometers were digitalized with 24-bit data acquisition system (Symmetric Research, Las Vegas, NV, USA) at a rate of $130 \mathrm{~Hz}$ and uploaded hourly to a cloud data storage site for offline processing. Hourly data was downloaded to a personal computer and transformed into successive thirty-second length segments for each of whom the power spectral density (PDS) was calculated. These hourly PDS segments were averaged together and the sum of them in $0-65 \mathrm{~Hz}$ frequency range was calculated over the study period. The local Earth's magnetic field was analized in five frequency ranges that overlap with the EEG ranges $[\mathrm{Hz}]$, but to differentiate those frequency ranges are obtained from the magnetic field in same range as the Schumann resonances occur we add an S in front: (SDelta) [0; 3.5], (STheta) [3.5; 7], (SAlpha) [7; 15], (SBeta) [15; 32], (SGamma) [32; 65]. We added [0; 65] where all frequency intervals were summed up in one to observe correlations in summarised MF spectrum.

\subsection{Statistical analysis}

The statistical analysis was performed using the software package SPSS 20.0. Nonparametric Mann-Whitney U test was used for the comparison of two independent samples. Pearson correlation coefficient for the linear correlation between two variables was calculated. The level of $p<0.05$ was considered statistically significant.

\subsection{Spectral analysis of the magnetometer data}

Consider magnetic field intensity $\left\{I_{t}\right\}_{t=0}^{N-1}$, where $t$ is discrete time variable:

$f(\omega)=\sum_{t=0}^{N-1} I_{t} \cdot e^{\frac{-2 \pi i t \omega}{N}}, \quad t \in Z$.

In order to transform $\left\{I_{t}\right\}_{t=0}^{N-1}$ to the frequency domain the discrete Fourier transform (DFT) (Eq. (1)) was used [19]. The drawback of DFT is that one cannot observe the change in spectral density over time unless sequentially computing DFT. To achieve this the discrete time short time Fourier transform (STFT) was employed:

$F(\tau, \omega)=\sum_{t=-\infty}^{\infty} I_{t} \cdot \xi(t-\tau) e^{-i t \omega}, \quad t \in Z$.

STFT for $\left\{I_{t}\right\}_{t=0}^{N-1}$ is represented by Eq. (2). In fact, this is essentially the analogue for Eq. (1) but applied to the function $I_{t} \cdot \xi(t-\tau) . \xi(t)$ is a so-called windowing function which has a value close to 1 in a subdomain of $t$ centered on 0 and a value close to 0 elsewhere. The units of $f(\omega)$ and $F(\tau, \omega)$ are $\mathrm{pT} \cdot \mathrm{s}$ due to the fact that the intensity of the magnetic field is measured in $\mathrm{pT}$ :

$S(\tau, \omega)=|F(\tau, \omega)|^{2}$.

Spectrograms investigated in this work is the squared modulus of STFT (Eq. (3)). Originally units of a spectrogram would be $\mathrm{pT}^{2} \cdot \mathrm{s}^{2} . S(\tau, \omega)$ is often referenced as power spectral density. Thus, the value of $S(\tau, \omega)$ is interpreted as signal power at the time interval $\Delta \tau$ and at the frequency range $\Delta \omega$.

More detailed algebraic and spectral analysis of local magnetic field intensity is presented in article [16].

\section{Results}

Significant correlations between weekly admissions of ACS cases and the average weekly 
TVMF strength in five frequency ranges was found. In the analyses of the whole year model two positive weak and moderate correlation coefficients in (SBeta) $[15 ; 32](r=0.06, p=0.65)$ and (SGamma) [32; 65] $(r=0.27, p=0.05)$ ranges were found in the female group, respectively. This means that a higher TVMF in SBeta and SGamma ranges is associated with higher number of ACS cases through the year in females. In males there was no significant correlations in analyses of the whole year model. The correlations between gender and weekly admissions of ACS and the TVMF changes within the year are presented in Fig. 4.

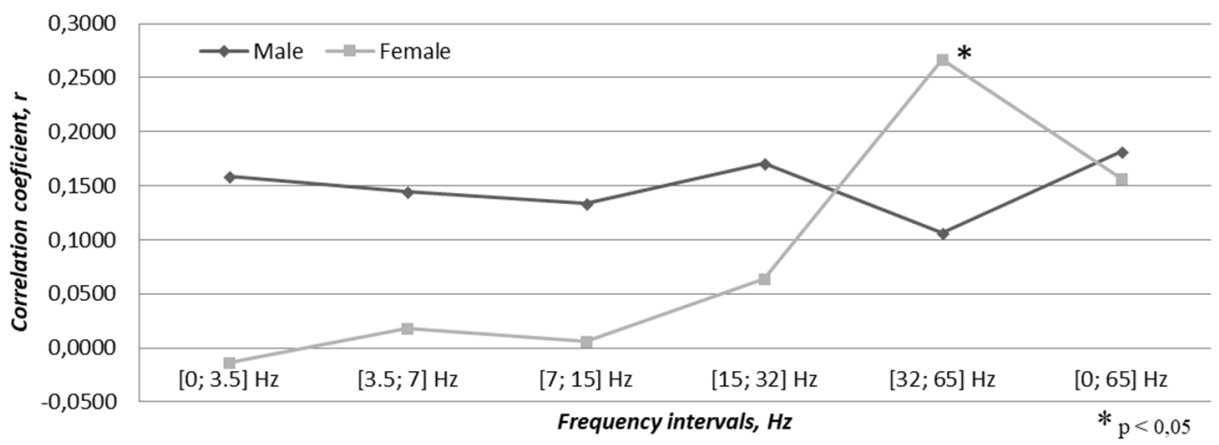

Fig. 4. Correlation between gender related cases of acute coronary syndrome and the time varying magnetic field changes through the year

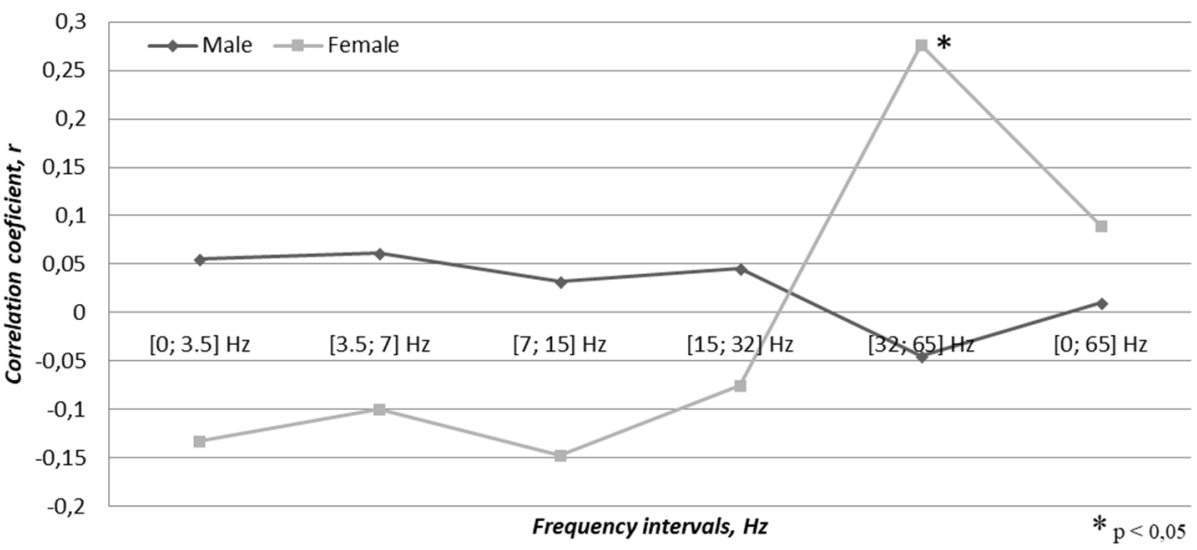

Fig. 5. Correlation between gender related cases of acute coronary syndrome and the time varying magnetic field changes through the first-half of the year

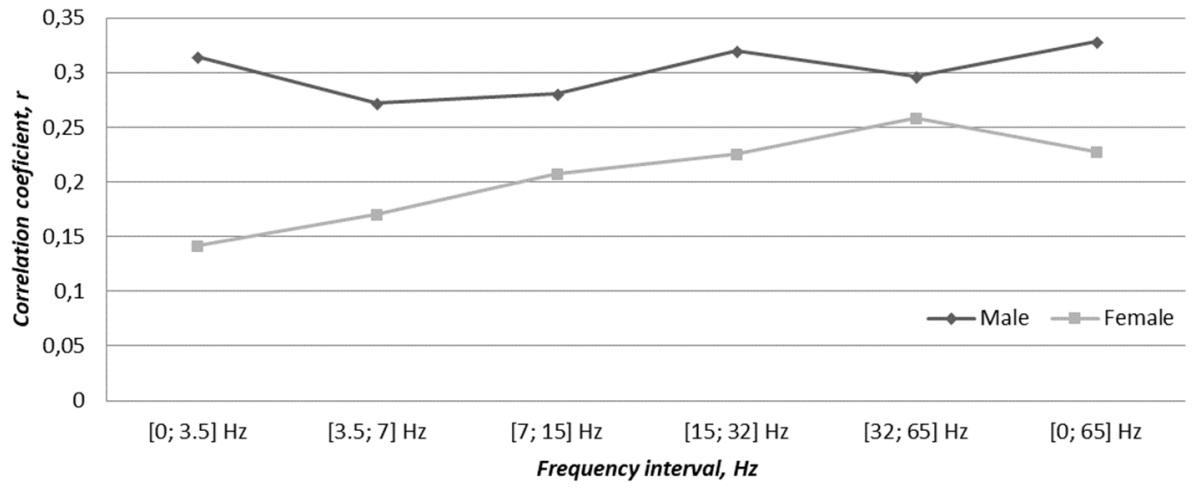

Fig. 6. Correlation between gender related cases of acute coronary syndrome and the time varying magnetic field changes through the second-half of the year 
When we divided the one-year period into two-time intervals, further analyses revealed that the first-half of the year had the same tendencies in the females with a single positive moderate correlation coefficient in (SGamma) $[32 ; 65]$ range $(r=0.28, p=0.05)$ (Fig. 5.). In males there were tendencies towards weak to moderate positive correlation coefficients $(p<0.1)$ at all frequency ranges in the second-half of the year, which are detailed in Fig. 6. There were no differences between ACS cases in male and female though the second-half of the year. The higher TVMF in SGamma range was associated with higher number of ACS cases through the year in females and through the second-half of the year in males.

Analyses based on specific CA lesions revealed significant correlation between acute coronary syndromes caused by LMA lesion and the TVMF changes. There were 46 males and 22 females with significant LMA lesions through the year. In males there were significant positive moderate correlation coefficients: (SDelta) $[0 ; 3.5](r=0.353, p=0.01)$, (STheta) $[3.5 ; 7](r=0.366$, $p=0.008)$, (SAlpha) [7; 15] $(r=0.339, p=0.014)$, (SBeta) $[15 ; 32](r=0.357, p=0.009)$ while in female there was a single positive and statistically significant moderate correlation coefficient in (SGamma) $[32 ; 65](r=0.430, p=0.001)$ through the year. That mean, that the stronger MF in SDelta, STheta, SAlpha and SBeta rangers is associated with higher number of ACS caused by LMA lesion in male, but the stronger MF in SGamma range is associated with higher number of ACS caused by LMA lesion in female through the year. These correlations are presented in Fig. 7.

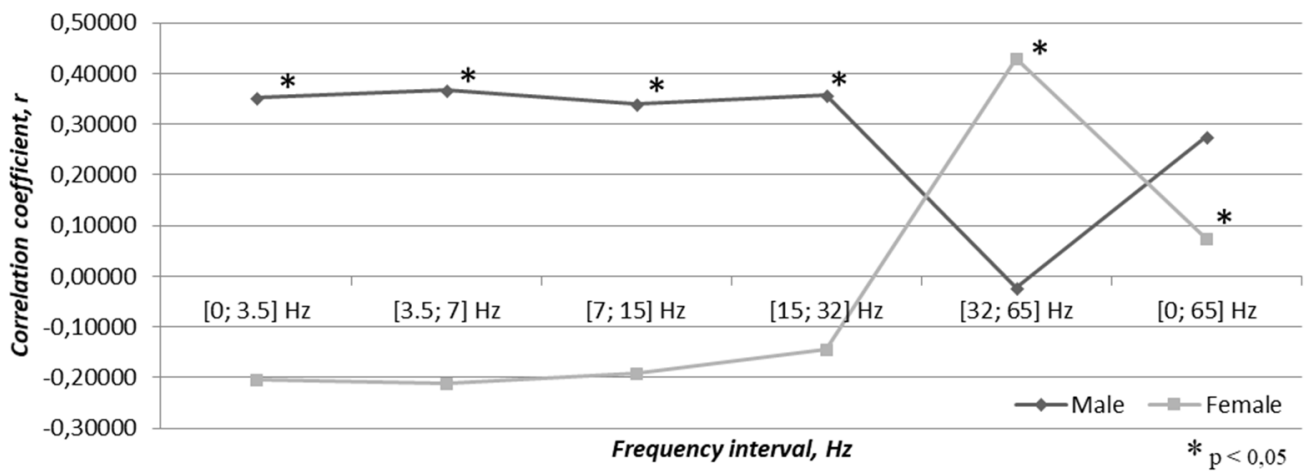

Fig. 7. Correlations between gender related cases of acute coronary syndrome caused

by left main artery lesion and the time varying magnetic field changes through the year

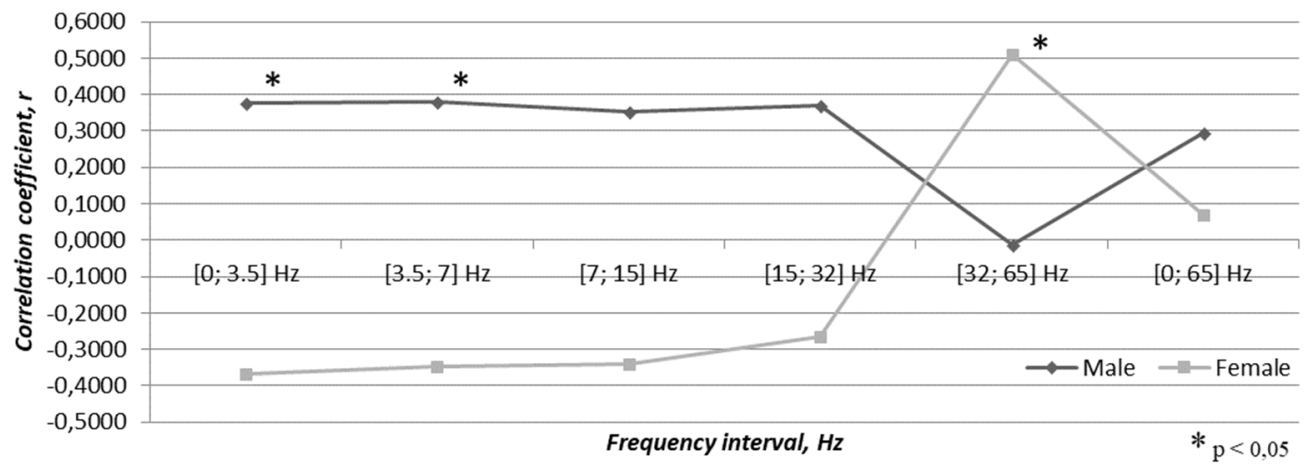

Fig. 8. Correlations between gender related cases of acute coronary syndrome caused by left main artery lesion and the time varying magnetic field changes through the first-half of the year

When analysed two half of the year model, general tendencies in the first-half of the year remain the same as in the whole year model, but due to low number of cases $p$-value was a bit higher. Statistically significant moderate correlation coefficients were found in (SDelta) [0; 3.5] 
$(r=0.3754, p=0.05)$ and (STheta) $[3.5 ; 7](r=0.3814, p=0.05)$ in male, while in female the tendencies remain the same with not decreased statistical significance in (SGamma) $[32 ; 65]$ $(r=0.5106, p=0.008$ ) (Fig. 8). That let us claim, that female has higher sensitivity to MF in SGamma range and the stronger MF in this range is potentially associated with higher rate of hospital admission due to LMA lesion caused ACS in female. There were not found statistically significant correlations in the second-half of the year due to low number of cases in groups.

\section{Discussion}

We believe that the effect on human regulatory systems significantly differs between the Global and the local Earth magnetic field. In contrary to previous studies we analysed the local Earth magnetic field changes (not the Global one) impact on manifestation of unstable angina pectoris. The correlations between weekly prevalence of ACS did not surprise us, as even stronger correlations were found in previous study on acute myocardial infarction relationship with changes of the TVMF [4]. In addition, this study demonstrated strong correlations between changes in the TVMF and manifestation of unstable angina pectoris caused by LMA lesion. Different sensitivity through the first and the second half of the year and through the whole year cannot be explained by general physiological principles and requires deeper understanding of processes occurring in the cells regulatory mechanisms. Significant differences between ACS caused by LMA lesion were observed in male and female. The ranges in which significant correlations were found in male were not significantly correlated in female and vice versa in female: the only range in which statistically significant correlation was found was the one for males did not prove significance. This suggests, that male and female have different sensitivity to the different ranges of the MF spectrum. In addition, further studies on MF differences between these periods of time might be necessary. We believe that it is important to compare correlations with cases of ACS in different frequency rates, as our study demonstrated different sensitivity rates within the spectrum of the MF.

Some interesting studies on lunar activity and occurrence of ACS have been published $[17,18]$. Sha et al. [17] found that in contrary to public opinion ACS is more frequent during new moon compared to full moon phases. This was strengthened by Ooman et al. [18] in study where significant differences between hospital admissions due to ACS on new moon versus full moon phases were presented. This study showed that more ACS cases occur within the period of new moon. Even more, the occurrences of cardiopulmonary resuscitations did not revealed relationship with the lunar activity [19]. This suggests that different medical conditions have different sensitivity to lunar activity and possibly other environment conditions. It is reported interesting case of the blind man, who had cyclical sleep-wake disorders. The man's circadian rhythm was based more on motion of the moon rather the sun [20]. Study conducted by Kanth et al. failed to prove the relationship between lunar phase and prevalence of ACS [21]. But previously reported human regulatory systems changes in accordance with the changes of lunar and solar activity are obvious and initiate hypothesis for further studies that lunar activity which possibly differs in the first and the second half of the year impacts prevalence of ACS. Due to patients' disturbances dividing them into lunar phase and period of the year much bigger sample size is necessary for significant conclusion in further studies.

Even more, the impact of temperature changes on number of acute myocardial infarction cases has been reported as well as other disease like rheumatic diseases, arterial hypertension, stroke and intraocular hypertension [22]. Some studies proposed that a temperature raise effects in increased prevalence of nonfatal ACS [23, 24]. Moreover, the Arctic Oscillation (AO) as a predictor of the weather temperature and humidity has been found as factor increasing the prevalence of both fatal and nonfatal ACS [25]. There is no doubt that environment status interfaces with human health condition and have impact on the course of chronic disease. An inclusion of the presence of chronic disease may add benefit to further studies on more reliable outcomes. 


\section{Limitations of the study}

Our study has some limitations. First, we included only patients admitted to our hospital omitting ones admitted to other hospitals in Kaunas and in other regions. Second, patients with symptomatic unstable angina pectoris but absent severe coronary stenoses were included as well, without taking into consideration the significance of the CA lesion. Third, concomitant illnesses and biochemical status were not taking into consideration, but they might be affected by MF changes as well and may burden the course of the main disease. Further studies counting concomitant illnesses and biochemical blood parameters in addition to severity of CA lesion is necessary.

\section{Conclusions}

1) Significant correlation between acute coronary syndrome and the local Earth magnetic field changes was revealed.

2) The acute coronary syndrome is positively correlated with the local Earth magnetic field in SGamma range in female through the year.

3) The acute coronary syndrome at all frequency intervals is positively correlated only in second-half of the year in male.

4) A strong and significant association between the stronger magnetic field in SDelta, STheta, SAlpha and SBeta rangers and higher number of acute coronary syndrome caused by the left main artery lesion in male through the year was found.

5) The stronger magnetic field in SGamma range is associated with higher number of acute coronary syndrome caused by left main artery lesion in female through the year.

6) The stronger magnetic field in SGamma range was associated with lower number of acute coronary syndrome caused by left main artery lesion in male.

7) The stronger magnetic field in SGamma range was the only one associated with higher number of acute coronary syndrome in female.

\section{Acknowledgements}

Thanks to the Head of the Cardiology department of the Hospital of Lithuanian University of Health Sciences Kaunas clinics for permission for the study implementation.

\section{References}

[1] Santos Gallego C.-G., Picatoste B., Badimón J. J. Pathophysiology of acute coronary syndrome. Current Atherosclerosis Reports, Vol. 16, 2014, p. 401.

[2] Makki N., Brennan T. M., Girotra S. Acute coronary syndrome. Journal of Intensive Care Medicine, Vol. 30, Issue 4, 2013, p. 186-200.

[3] Hedayat K. Systems Biology Research Group. 2017, https:/www.facebook.com/plugins/post.php ?href=https $\% 3 \mathrm{~A} \% 2 \mathrm{~F} \% 2 \mathrm{Fwww}$.facebook.com\%2Fsystemsbiologyresearchgroup $\% 2 \mathrm{Fposts} \% 2 \mathrm{~F} 30020$ $4793813215 \&$ width $=500$.

[4] Jaruševičius G., Rugelis T., Mccraty R., Landauskas M., Berškienė K., Vainoras A. Correlation between changes in local Earth's magnetic field and cases of acute myocardial infarction. International Journal of Environmental Research and Public Health, Vol. 15, 2018, p. 399.

[5] Liboff A. R. A role for the geomagnetic field in cell regulation. Electromagnetic Biology and Medicine, Vol. 29, 2010, p. 105-112.

[6] Alabdulgader A., Mccraty R., Atkinson M., Vainoras A., Berškienė K., Mauricienė V., Daunoravičienė A., Navickas Z., Šmidtaitė R., Landauskas M. Human heart rhythm sensitivity to earth local magnetic field fluctuations. Journal of Vibroengineering, Vol. 17, 2015, p. 3271-3278.

[7] Gaetani R., Ledda M., Barile L., Chimenti I., De Carlo F., Forte E., Ionta V., Giuliani L., D'emilia E., Frati G. Differentiation of human adult cardiac stem cells exposed to extremely low-frequency electromagnetic fields. Cardiovascular Research, Vol. 82, 2009, p. 411-420. 
[8] Gurfinkel Y. I., At'kov O. Y., Vasin A. L., Breus T. K., Sasonko M. L., Pishchalnikov R. Y. Effect of zero magnetic field on cardiovascular system and microcirculation. Life Sciences in Space Research, Vol. 8, 2016, p. 1-7.

[9] Gmitrov J., Gmitrova A. Geomagnet field effect on cardiovascular regulation. Bioelectromagnetics, Vol. 25, 2004, p. 92-101.

[10] Garcia R., Sosner P., Laude D., Hadjadj S., Ragot S. Spontaneous baroreflex sensitivity measured early after acute myocardial infarction is an independent predictor of cardiovascular mortality: results from 12-year follow-up study. International Journal of Cardiology, Vol. 177, 2014, p. 120-122.

[11] Weydahl, Sothern R. B., Cornelissen G., Wetterberg L. Geomagnetic activity influences the melatonin secretion at latitude 70 degrees. Biomedicine and Pharmacotherapy, Vol. 55, 2001, p. 57-62.

[12] Burch J. B., Reif J. S., Yost M. G. Geomagnetic activity and human melatonin metabolite excretion. Neuroscience Letters, Vol. 438, 2008, p. 76-79.

[13] Hardell L. World Health Organization, radiofrequency radiation and health - a hard nut to crack (Review). International Journal of Oncology, Vol. 51, 2017, p. 405-413.

[14] Pall M. L. Microwave frequency electromagnetic fields (EMFs) produce widespread neuropsychiatric effects including depression. Journal of Chemical Neuroanatomy, Vol. 75, 2016, p. 43-51.

[15] Huss A., Peters S., Vermeulen R. Occupational exposure to extremely low-frequency magnetic fields and the risk of ALS: A systematic review and meta-analysis. Bioelectromagnetics, Vol. 39, 2018, p. $156-163$.

[16] Landauskas M., Vainoras A., et al. Algebraic and spectral analysis of local magnetic field intensity. Proceedings of the Lithuanian Mathematical Society, Vol. 56, 2015, p. 54-59.

[17] Sha L. R., Xu N. T., Song et al. X. H. Lunar phases, myocardial infarction and hemorrheological character. A Western medical study combined with appraisal of the related traditional Chinese medical theory. Chinese Medical Journal (English Edition), Vol. 102, 1989, p. 722-725.

[18] Oomman A., Ramachandran P., Shanmugapriya, et al. A novel trigger for acute coronary syndromes: The effect of lunar cycles on the incidence and in-hospital prognosis of acute coronary syndromes - a 3-year retrospective study. Journal of the Indian Medical Association, Vol. 101, 2003, p. $227-228$.

[19] Alves D. W., Allegra J. R., Cochrane D. G., Cable G. Effect of lunar cycle on temporal variation in cardiopulmonary arrest in seven emergency departments during 11 years. European Journal of Emergency Medicine, Vol. 10, 2003, p. 225-228.

[20] Miles L. E., Raynal D. M., Wilson M. A. Blind man living in normal society has circadian rhythms of 24.9 hours. Science, Vol. 198, 1977, p. 421-423.

[21] Kanth R., Berg R. L., Rezkalla Sh H. Impact of lunar phase on the incidence of cardiac events. World Journal of Cardiovascular Diseases, Vol. 2, 2012, p. 124-128.

[22] Messner T. Environmental variables and the risk of disease. International Journal of Circumpolar Health, Vol. 64, Issue 5, 2005, p. 523-533.

[23] De Senarclens C., Assimacopoulos A., Altherr J., et al. Myocardial infarction and meteorology. Schweizerische Medizinische Wochenschrift, Vol. 110, 1980, p. 1931.

[24] Miric D., Rumboldt Z. The impact of meteorological factors on the onset of myocardial infarction in the coastal region of middle Dalmatia. Giornale Italiano Di Cardiologia, Vol. 23, 1993, p. 655-660.

[25] Mcgregor G. R. Winter North Atlantic Oscillation, temperature and ischaemic heart disease mortality in three English counties. International Journal of Biometeorology, Vol. 49, 2005, p. 197-204. 